\title{
Studies of the Cosmic Infrared Background with the Space Infrared Telescope Facility (SIRTF)
}

\author{
M. W. Werner \\ Jet Propulsion Laboratory, California Institute of Technology, \\ Pasadena, CA, 91109, USA, \\ William T. Reach \\ SIRTF Science Center, California Institute of Technology, Pasadena, \\ $C A, 91125, U S A$ \\ M. Rieke \\ Steward Observatory, University of Arizona, Tucson, AZ, 85721, USA
}

\begin{abstract}
IRAS, COBE, and ISO have demonstrated the unique importance of a cryogenic infrared telescope in space for studying diffuse infrared backgrounds and for teasing out the individual point sources which contribute to them. This importance results from the extremely high infrared sensitivity of such telescopes, particularly to diffuse radiation. The next cryogenic infrared telescope will be NASA's Space Infrared Telescope Facility (SIRTF), which is currently in the final stages of construction leading to launch in 2002. SIRTF will be the first infrared space observatory to make extensive use - both for imaging and spectroscopy of large format infrared detector arrays. The sensitivity and spatial and spectral coverage of SIRTF's array-based instruments endow SIRTF with great power for the study of the cosmic infrared background (CIRB) and related scientific issues. This paper reviews the SIRTF mission design and measurement functionality and describes SIRTF's potential studies of the CIRB, drawing examples from the programs planned by the SIRTF Guaranteed Time Observers (GTO's). We also summarize the opportunities for community participation in SIRTF.
\end{abstract}

\section{SIRTF Overview}

\subsection{Mission Description}

The principal features of the SIRTF mission are summarized in Table 1; in addition, SIRTF and its instruments are described in a number of conference publications, the most recent being SPIE Symposium 4131, held in San Diego in the summer of 2000 (see also Fanson et al. 1998, and other papers in the Proceedings of SPIE Symposium 3356). For present purposes, therefore, we describe only the orbit and the novel thermal design it enables. 
SIRTF will operate from an Earth-trailing heliocentric orbit. A Delta $7920 \mathrm{H}$ rocket will launch SIRTF into this orbit in which it escapes from the Earth's gravity and joins the Earth in orbiting the Sun in an orbit of approximately $1 \mathrm{AU}$ semi-major axis. SIRTF drifts slowly away from the Earth, reaching a distance of about $0.62 \mathrm{AU}$ after 5 years. This orbit has several substantial advantages over the low- or near-Earth orbits used by previous astrophysical observatories. Chief among these is that the heat load of the Earth is never an issue. This enables the use of a novel warm launch architecture in which SIRTF is launched with the telescope warm and the instruments cold in a relatively small, liquid-helium cryostat directly behind the telescope (Figure 1). This Cryogenic Telescope Assembly sits atop a fairly conventional spacecraft. SIRTF uses a fixed solar panel which is always kept oriented towards the Sun and shields the thermally sensitive outer shell of the telescope-instrument system from direct sunlight. In turn, the anti-Sun surface of this shell is painted black and optimized to radiate away the small amount of heat the shell receives from the solar panel and the spacecraft. In the heliocentric orbit, the radiating surface is never illuminated by the heat of the Earth, which would seriously compromise its performance.

Following launch, the telescope cools from room temperature to its final operating temperature of $\sim 5.5 \mathrm{~K}$ by a combination of radiation to space and the cooling power of the helium vapor which escapes from the cryostat. This will take of order 30 days. At that point, the outer shell temperature equilibrates at about $31 \mathrm{~K}$, and the parasitic heat load into the cryostat is negligible, even in comparison to the very small instrument dissipation of about $4 \mathrm{~mW}$. Our current models of this high-performance cryogenic system suggest that a lifetime of order 5 years can be achieved with an initial cryogen load of $\sim 350$ liters. This is a dramatic improvement over the ISO mission, which achieved lifetime $\sim 2.5 \mathrm{yr}$ in near-Earth orbit with about 2400 liters of liquid helium. The warm-launch architecture separates the size of the cryostat and its attendant [necessarily massive] vacuum shells from that of the optical system. Thus, this approach points the way to future, larger infrared space observatories such as the NGST and SAFIR missions highlighted in the recent National Academy of Sciences decadal review of astronomical missions.

Table 1. SIRTF Mission Charecteristics

\begin{tabular}{lll}
\hline Lifetime & & 2.5 to $5 \mathrm{yr}$ \\
Aperture & & $85 \mathrm{~cm}$ \\
Orbit & & Heliocentric, $1 \mathrm{AU}$ \\
Diffraction limit & & $6.5 \mu \mathrm{m}$ \\
Image Size & $\simeq 1.5^{\prime \prime}$ \\
Pointing.... & accuracy & $5^{\prime \prime}(1 \sigma$, radial $)$ \\
& a posteriori knowledge & $1.4^{\prime \prime}(1 \sigma$, radial $)$ \\
& stability & $0.3^{\prime \prime}(1 \sigma$, radial $)$ \\
Telescope temperature & offset accuracy & $0.4^{\prime \prime}(1 \sigma$, radial $)$ \\
Wavelength range.... & imaging, mapping & $<5.5 \mathrm{~K}$ \\
& spectroscopy & $3-180 \mu \mathrm{m}$ \\
& spectral energy distribution & $5-40 \mu \mathrm{m}$ \\
Planetary tracking rate & & $53-100 \mu \mathrm{m}$ \\
& & up to $1.0^{\prime \prime} / \mathrm{s}$ \\
\hline
\end{tabular}




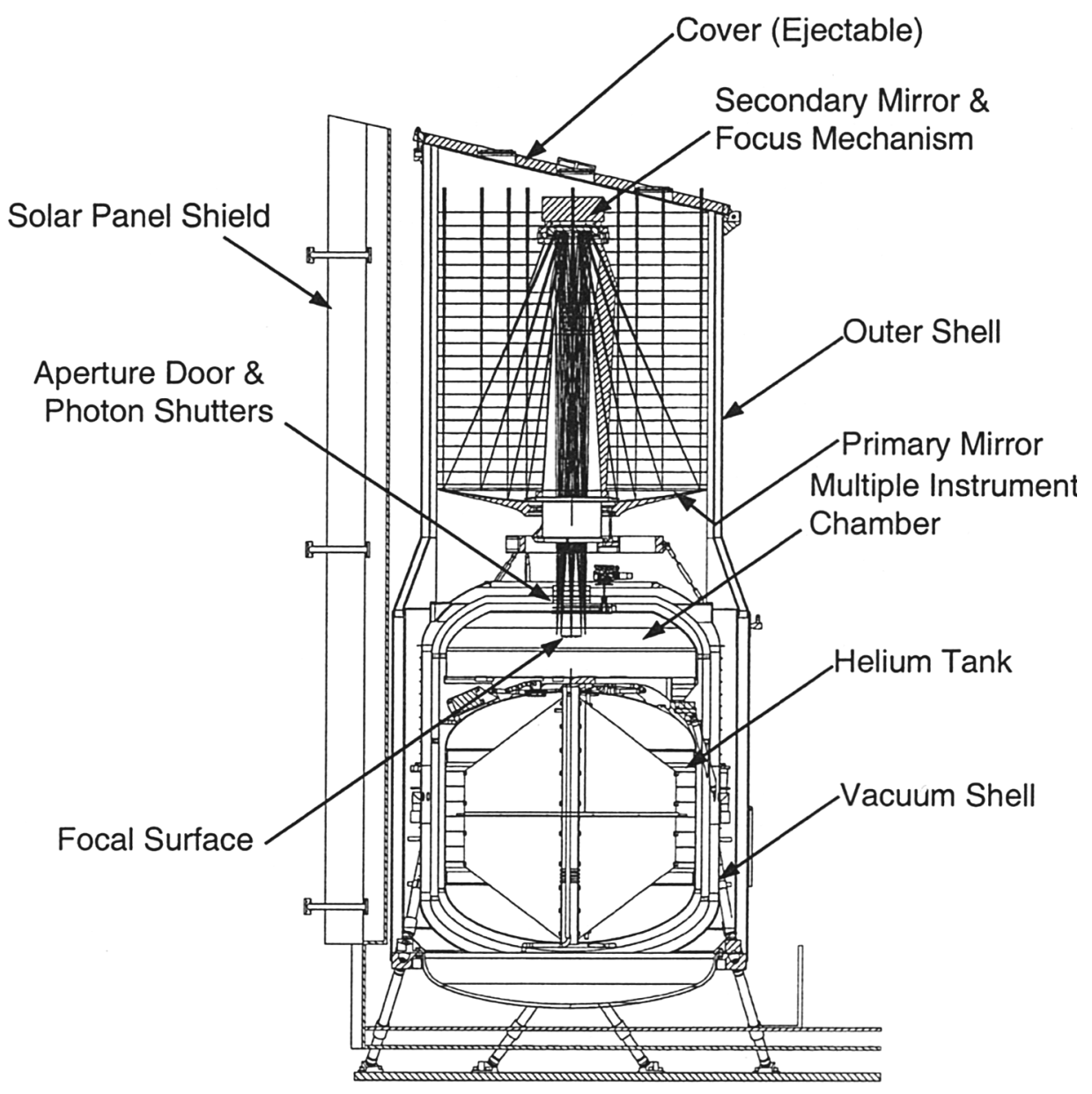

Figure 1. A cut-away view of the SIRTF Cryogenic Telescope Assembly, illustrating the warm launch architecture. Note the small cryogen tank below the telescope. In flight, the solar panel, which is just exterior to the solar panel shield at the left of the drawing, is kept oriented towards the Sun. The portion of the outer shell opposite the solar panel is blackened so that the outer shell cools radiatively to $<35 \mathrm{~K}$ in space. 


\subsection{Measurement Functionality}

The measurement functionality of SIRTF's three instruments is described below. Briefly, SIRTF provides imaging, photometry, and surveying in seven photometric bands (at 3.6, 4.5, 5.8, 8, 24, 70 and $160 \mu \mathrm{m}$ ), low resolution imaging spectroscopy $(R \sim 60-120)$ from $5.3-40 \mu \mathrm{m}$, and high resolution spectroscopy ( $R \sim 600)$ from $10-37 \mu \mathrm{m}$. In addition, SIRTF will carry out spectrophotometry from 53-100 $\mu \mathrm{m}$ with $(R \sim 15)$. All three of SIRTF's instruments use large-format infrared detector arrays. The six photometric bands between 3.6 and $70 \mu \mathrm{m}$ each use an array with a $5^{\prime} \times 5^{\prime}$ field of view. This provides SIRTF with very powerful capabilities for efficient, large area surveys which are of particular importance for resolving and counting the sources which may contribute to the CIRB. In addition, SIRTF's array-based spectrograph modules provide the diagnostic capabilities required to determine the physical character of such sources. The three instruments and their capabilities are:

The Infrared Array Camera (IRAC: Principal Investigator Giovanni Fazio, SAO) IRAC provides wide-field imaging over two adjacent $5^{\prime} \times 5^{\prime}$ fields of view. One is imaged at 3.6 and $5.8 \mu \mathrm{m}$, simultaneously via the use of a beamsplitter; the other is similarly imaged at 4.5 and $8 \mu \mathrm{m}$. InSb arrays with $256 \times 256$ pixels are used at 3.6 and $4.5 \mu \mathrm{m}$; Si:As IBC arrays with $256 \times 256$ pixels at 5.8 and $8 \mu \mathrm{m}$. Bandwidths are $\sim 25 \%(\sim 40 \%$ at $8 \mu \mathrm{m})$. Expected sensitivities at high galactic latitude (all quoted sensitivities are $5 \sigma$ in $500 \mathrm{~s}$ ) are $3,4,10$, and $15 \mu \mathrm{Jy}$ at $3.6,4.5,5.8$, and $8 \mu \mathrm{m}$, respectively.

The Multiband Imaging Photometer for SIRTF (MIPS: Principal Investigator George Rieke, Arizona) MIPS provides imaging and photometry in bands at 24,70 , and $160 \mu \mathrm{m}$, optimized either for efficient large area surveys or to provide excellent sampling of the Airy disk. The arrays are $128 \times 128$ pixel Si:As IBC at $24 \mu \mathrm{m}, 32 \times 32$ pixel Ge:Ga at $70 \mu \mathrm{m}$, and $2 \times 20 \mathrm{Ge}: \mathrm{Ga}$ (stressed) at $160 \mu \mathrm{m}$. The survey fields of view are $5^{\prime} \times 5^{\prime}$ at $24 \mu \mathrm{m}$ and $70 \mu \mathrm{m}$ and $5^{\prime} \times 0.5^{\prime}$ at $160 \mu \mathrm{m}$. Expected sensitivities $(5 \sigma$ in $500 \mathrm{~s})$ at high galactic latitude are $0.2,1.5$, and $30 \mathrm{mJy}$ at 24,70 , and $160 \mu \mathrm{m}$, respectively. The $160 \mu \mathrm{m}$ sensitivity is limited at this level by confusion due to extragalactic sources. In addition, the MIPS provides spectrophotometry with resolving power $\sim 15$ and sensitivity $\sim 10 \mathrm{mJy}$ over the $53-100 \mu \mathrm{m}$ band.

The Infrared Spectrograph (IRS: Principal Investigator James Houck, Cornell University) IRS is a modular instrument with four separate spectrograph modules. The two low resolution modules provide long slit imaging spectroscopy from $5.3-14 \mu \mathrm{m}$ and $14-40 \mu \mathrm{m}$, respectively. Typical sensitivities ( $5 \sigma$ in $500 \mathrm{~s}$ ) are expected to be $\sim 0.5 \mathrm{mJy}$ in the shorter wavelength module and $\sim 1.5 \mathrm{mJy}$ in the longer wavelength module. The resolving power is in the range 60-120 for these modules. The two high-resolution (resolving power 600) modules cover the wavelength ranges $10-19.5 \mu \mathrm{m}$ and $19.5-37 \mu \mathrm{m}$. Each operates in echelle mode so that the entire spectral octave is covered in each spectrum. Projected emission line sensitivites are $\sim 3 \times 10^{-18} \mathrm{~W} \mathrm{~m}^{-2}$ for each module. Each of the four modules is equipped with a Si:xx IBC array in $128 \times 128$ format. The dopant is As for the two shorter wavelength modules and $\mathrm{Sb}$ for the longer wavelengths. 


\subsection{The Zodiacal Ring}

The results from IRAS and COBE show that, in the infrared, the zodiacal emission is not perfectly smooth but includes structures on a variety of spatial scales. These include not only the dust rings and dust trails due, respectively, to asteroid collisions and the accumulation of cometary ejecta, but also an Earthtrailing enhancement which results from gravitational focussing of dust which is spiraling sunwards under the Poynting-Robertson effect (Dermott et al. 1994; Reach et al. 1995). Proper modelling of this and similar dynamic phenomena is an important prerequisite to looking beyond the solar system at Galactic and extragalactic infrared backgrounds. In addition, such enhancements may either masquerade as, or signal the presence of, extra-solar planets when observed by future missions seeking to image planets around nearby stars. As shown by Jayarman, Dermott, and Werner (1996), SIRTF's Earth-trailing heliocentric orbit will carry the spacecraft towards and, eventually, directly through this enhancement, providing us the opportunity to observe it from a variety of unique vantage points. These in situ observations will directly test our models of the formation and persistence of such enhancements.

\subsection{The SIRTF Legacy Program}

SIRTF, like NASA's other major observatories, is accessible to the entire international scientific community through the usual peer reviewed proposal process. The first solicitation of proposals for time on SIRTF was the Call for Legacy Science Proposals, issued at the end of June, 2000. Legacy Science projects are large investigations aimed at exploiting SIRTF's unique capabilities, at creating a significant scientific legacy in the form of scientific publications and archival data products, and at encouraging follow-on observations with SIRTF during the mission's limited lifetime. Consistent with this latter objective, the pipeline-processed data are to be released publicly via the SIRTF archive at the same time they are passed on to the team executing the program. An individual Legacy project is expected to utilize hundreds if not thousands of hours of SIRTF observations, and upward of 3000 total hours will be dedicated to this program during the early phases of the SIRTF science mission. The selected Legacy teams and their projects will be announced in November, 2000, and it is possible that one or more of those projects will be closely related to the Cosmic Infrared Background.

\section{GTO Surveys}

Many approaches to the study of the CIRB will require unbiased surveys - both to estimate the integrated background due to discrete sources and to identify specific targets for detailed study to illuminate the nature of these sources. SIRTF, with its large area arrays, large fields of view, and very high sensitivity, is very well-suited to carrying out photometric/imaging surveys of this type, using the IRAC from 3.6-8 $\mu \mathrm{m}$ and the MIPS from 24-160 $\mu \mathrm{m}$. SIRTF's capabilities in this area can best be illustrated by describing the extragalactic surveys planned by the Guaranteed Time Observers (GTOs), as shown in Table 2. The GTOs have adopted a nested survey strategy in which large areas are to be done at 
relatively low sensitivity, while smaller areas are identified for much deeper observations. Similar surveys covering greater or lesser areas of the sky may be included in the Legacy Science program, and other investigations of this type might be proposed in response to the General Observer opportunities discussed below. Also shown in Table 2 is the First Look Survey (FLS) planned for execution by the SIRTF Science Center at the very start of the mission. Although the FLS does not go as deep as the GTO surveys, it should be noted that the data from the FLS will be released via the SIRTF data archive as soon as they can be validated, certainly well in advance of the first post-launch call for SIRTF General Observer proposals.

Table 2. Extragalactic Surveys Planned with SIRTF

\begin{tabular}{|c|c|c|c|c|c|c|c|c|}
\hline \multirow[t]{2}{*}{ Type } & \multirow[t]{2}{*}{$\begin{array}{l}\text { Area } \\
\text { deg}^{2}\end{array}$} & \multicolumn{7}{|c|}{$\begin{array}{c}\text { 5- } \sigma \text { limiting flux, } \mu \mathrm{Jy} \\
\text { (5- } \sigma \text { limiting magnitude) }\end{array}$} \\
\hline & & $3.6 \mu \mathrm{m}$ & $4.5 \mu \mathrm{m}$ & $5.8 \mu \mathrm{m}$ & $8 \mu \mathrm{m}$ & $24 \mu \mathrm{m}$ & $70 \mu \mathrm{m}$ & $160 \mu \mathrm{m}$ \\
\hline Wide $^{a}$ & 9 & $\begin{array}{l}8.4 \\
18.7\end{array}$ & $\begin{array}{c}11 \\
17.8\end{array}$ & $\begin{array}{c}3 \\
16.3\end{array}$ & $\begin{array}{c}38 \\
15.5\end{array}$ & 600 & 3600 & 33000 \\
\hline Deep $^{b}$ & $\overline{2}$ & $\begin{array}{l}2.5^{c} \\
20.1\end{array}$ & $\begin{array}{l}3.6 \\
19.0\end{array}$ & $\begin{array}{l}11.4 \\
17.4\end{array}$ & $\begin{array}{l}15.3 \\
16.5\end{array}$ & $\overline{150}$ & 1000 & $30000^{c}$ \\
\hline $\begin{array}{c}\text { Real } \\
\text { Deep }^{d}\end{array}$ & 0.2 & $\begin{array}{l}2.5^{c} \\
20.1\end{array}$ & $\begin{array}{l}2.5^{c} \\
19.4\end{array}$ & $\begin{array}{l}2.5 \\
19.1\end{array}$ & $\begin{array}{c}3.4 \\
18.1\end{array}$ & $30^{c, f}$ & $700^{c, f}$ & \\
\hline $\begin{array}{l}\text { First } \\
\text { Look }^{e}\end{array}$ & $\overline{5}$ & $\begin{array}{c}23 \\
17.6\end{array}$ & $\begin{array}{c}24 \\
17.0\end{array}$ & $\begin{array}{l}70 \\
15.5\end{array}$ & $\begin{array}{c}58 \\
15.0\end{array}$ & 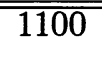 & $\overline{3800}$ & $\begin{array}{l}33000 \\
\end{array}$ \\
\hline
\end{tabular}

${ }^{a}$ NOAO deep field

${ }^{b}$ HDF-N, Groth strip, CXO-S, SSA 13, Lockman Hole, XMM Deep

${ }^{c}$ sensitivity estimated to be limited by confusion due to extragalactic sources

${ }^{d}$ Groth strip

$e$ North ecliptic pole

${ }^{f}$ MIPS Real Deep survey will cover only $0.02 \mathrm{deg}^{2}$

Several points about the surveys shown in Table 2 are noteworthy and will be explored further below. Firstly, the combination of sensitivity and area covered will make these types of surveys a much more powerful probe of the early Universe than were earlier surveys either by IRAS or by ISO.

Secondly, coordinated imaging surveys are planned across the SIRTF wavelength range of 3.6 to $160 \mu \mathrm{m}$, so that complete spectral energy distributions will be obtained for many targets, while in other cases even the absence of detections at certain wavelengths will support determinations of photometric redshifts. This multiwavelength coverage will allow SIRTF to bridge the gap between wavelengths dominated by direct photospheric light and those dominated by dust reradiating absorbed starlight.

Thirdly, note that the fields identified by the GTOs for survey observations include fields which will be well-studied in many other spectral bands, both from space and from the ground. This is particularly the case for the Deep Survey fields, a series of $\sim 0.5 \operatorname{deg}^{2}$ areas which include or cover such regions as the Groth Strip, the Northern Hubble Deep Field, and the Southern Chandra X-Ray Observatory deep field. 


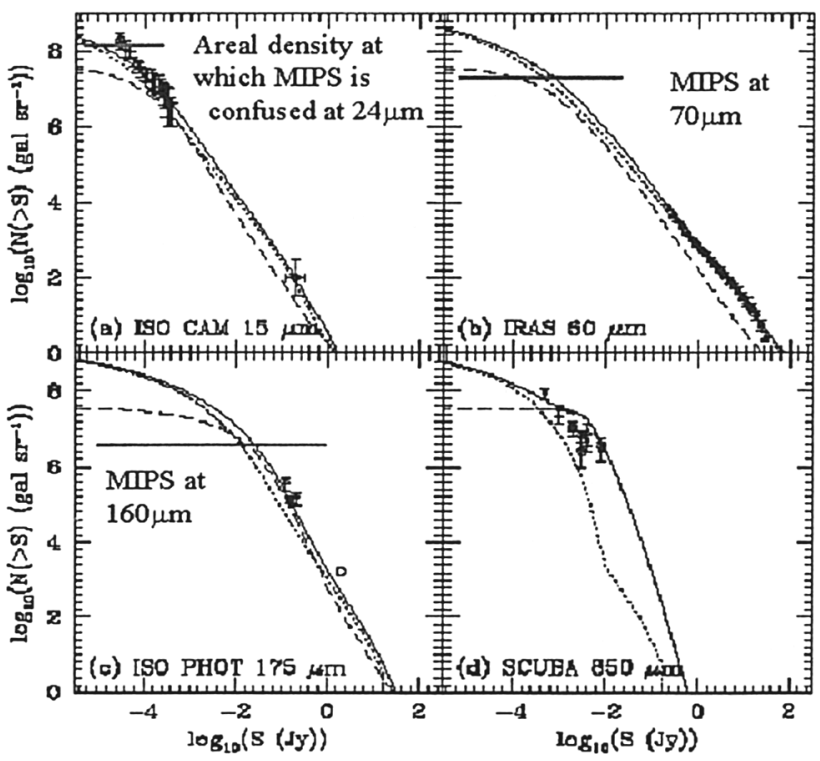

$=$ source density on the sky at which MIPS will be confusion-limited (10 beams/source)

Figure 2. Source counts from ISO, IRAS, and SCUBA are displayed, together with the source densities at which SIRTF will be confusion limited. The theoretical curves are taken from Tan et al. (1999).

\section{SIRTF's Contributions to the Studies of the Cosmic Infrared Background}

We describe some specific areas where the results of the surveys shown in Table 2 , as well as other investigations from SIRTF, will contribute to the study of the cosmic infrared background.

\subsection{Source Counts}

Source counts to various limiting fluxes will be an important product of SIRTF's surveys. Consider first the far infrared wavelengths, where the detection of a CIRB at wavelengths longward of $100 \mu \mathrm{m}$ has been reported based on the COBE data. At $70 \mu \mathrm{m}$ (Fig. 2), SIRTF will extend the source counts to $\sim 100 \times$ fainter flux levels than has been possible to date; the improvement in sensitivity at $160 \mu \mathrm{m}$ will be about an order of magnitude. At somewhat shorter wavelengths where SIRTF's sensitivity gain over the faintest ISO surveys is no more than an order of magnitude, SIRTF will achieve this sensitivity over much larger areas of the sky than did ISO. As is shown in Figure 2a, for example, SIRTF's ultradeep $24 \mu \mathrm{m}$ survey will reach an areal density of order $10^{8}$ galaxies/sr, comparable to that achieved by ISOCAM's deepest $15 \mu \mathrm{m}$ survey. However, the SIRTF survey will cover at least $0.2 \mathrm{sq}$ degree, much more if warranted by the results, while the ISO results are based on observations of less than one-twentieth of this area. As is shown in Figure 3, source counts from SIRTF to the levels indicated in Figure 2 can resolve a substantial fraction (typically of order $50 \%$ ) 


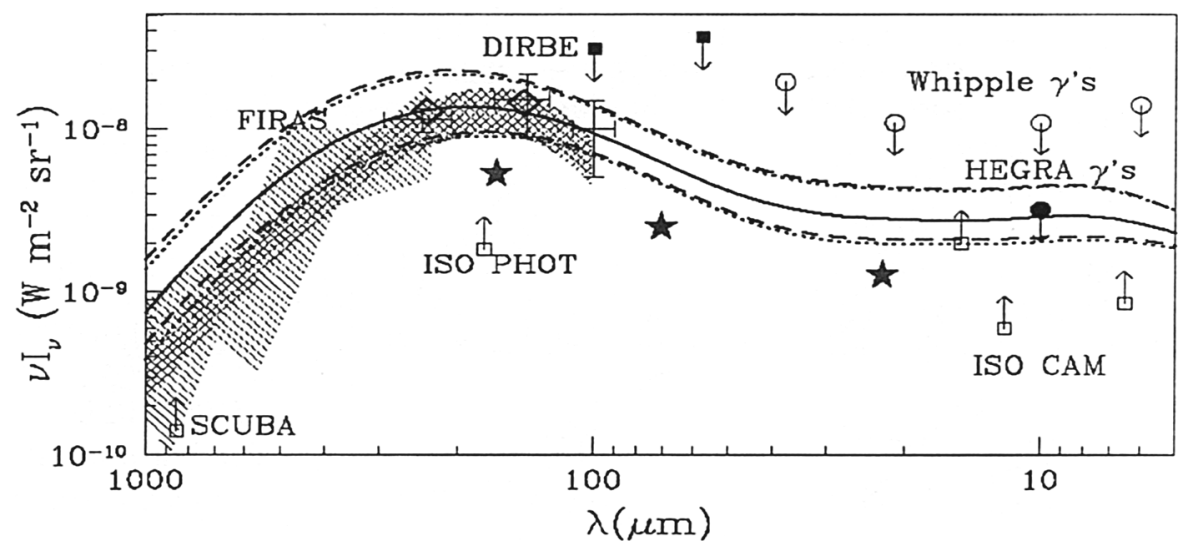

Figure 3. Observations and predictions of the cosmic infrared background, taken from Tan et al. 1999. The three stars at 24, 70, and $160 \mu \mathrm{m}$ show the integrated flux attributable to discrete sources measurable by SIRTF according to the predicted number counts and confusion limits shown in Figure 2.

of the long wavelength infrared background expected from the models of Tan, Silk, \& Belland (1999). This is also clear from Figure 2, as in each case the SIRTF measurements will extend below the flux level at which the predicted source counts turn over.

SIRTF can make equally important contributions to the determination of the background at short wavelengths from 3.5 to $8 \mu \mathrm{m}$. Consider what would be required to track down the sources responsible for the extragalactic $3.5 \mu \mathrm{m}$ background $\sim 8 \mathrm{kJy} / \mathrm{sr}$ reported from DIRBE measurements by Dwek \& Arendt (1998) and by Gorjian, Wright, \& Chary (2000). Firstly, SIRTF's sensitivity will facilitate removal of the contributions from galactic stars at these wavelengths, as has been done by Wright (2000) at $2.2 \mu \mathrm{m}$ using the 2MASS data base, perhaps improving on the background estimate quoted above. Then, as is shown in Figure 4, SIRTF's galaxy counts at $3.5 \mu \mathrm{m}$ will reach flux levels hundreds of times fainter than those previously attained and extend below the flux level at which the predicted source counts turn over at this wavelength as well. More specifically, if the model used in Figure 4 is correct, the integrated flux of the galaxies detected by SIRTF would be $\sim 2 \mathrm{kJy} / \mathrm{sr}$, so that the SIRTF observations would resolve $\sim 25 \%$ of the reported background flux while suggesting that the remainder might not be attributable to the galaxy population seen by SIRTF. This discussion presumes a correction for zodiacal foreground based on DIRBE. Although SIRTF will not be able to match DIRBE's systematic study of the zodiacal light, there may be ways of using SIRTF to correct for the zodiacal contribution to its own measurements, following the approach outlined by Wright, Werner, \& Rieke (1995).

SIRTF will be able to use other techniques to probe still deeper than indicated by the confusion limits presented above. One is to use the magnification of background sources provided by foreground gravitationally-lensing galaxy clus- 


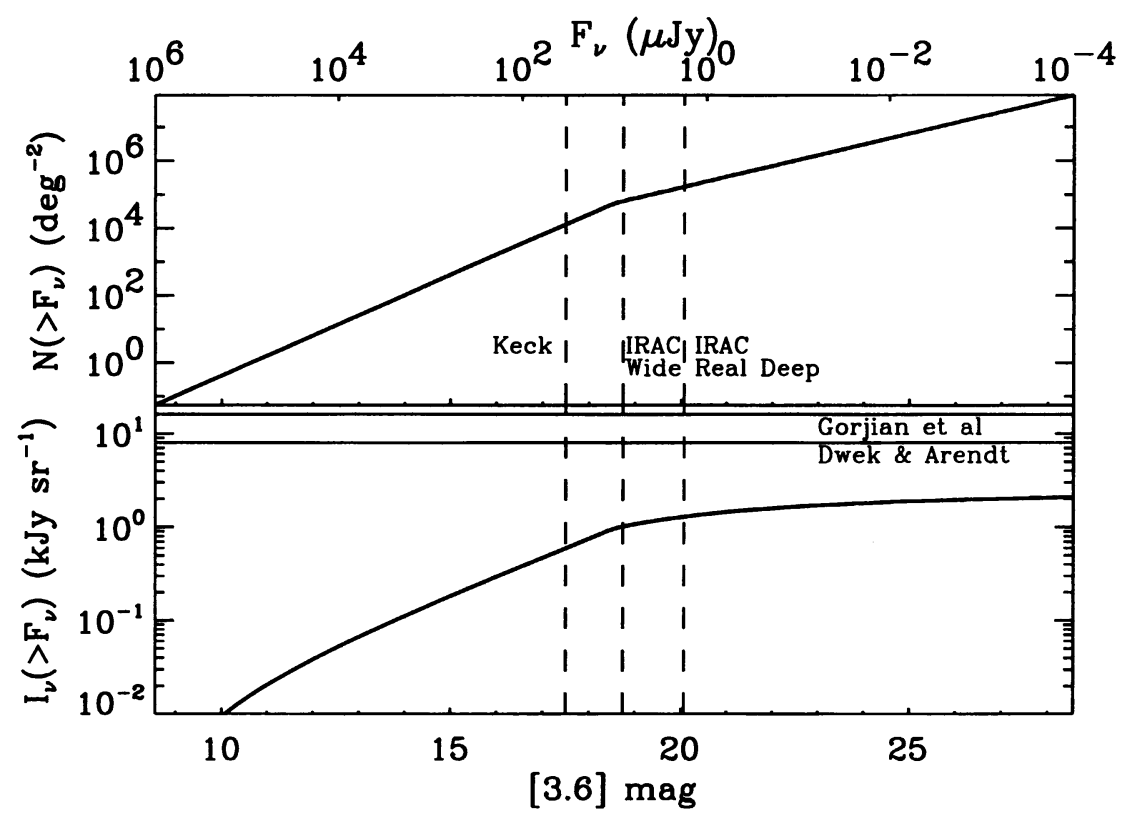

Figure 4. Predicted galaxy source counts at $3.6 \mu \mathrm{m}$, taken from Franceschini et al. (1991, Fig. 5). The vertical lines show the flux levels reached in the limited area Keck survey by Hogg et al. (2000) as well as those to be achieved in the "Wide" and "Real Deep" IRAC surveys summarized in Table 2 .

ters to reach flux levels fainter than those set by confusion in unlensed regions of the sky, as discussed by L. Metcalfe elsewhere in this volume. A second technique would be to obtain accurate measurements of the fluctuations of the sky brightness in reaching below the confusion limit for comparison with models based on assumed source distributions.

\subsection{Bridging the Gap, or How Much Star Formation is Hidden in the Infrared?}

SIRTF's broad spectral coverage and high sensitivity across the infrared band will bridge the gap between the visible and near infrared explorations carried out from HST and from the ground and the exciting deep surveys carried out in the submillimeter using the SCUBA instrument on the JCMT. As suggested by Figure 2, the confusion-limited source areal density of the SCUBA surveys would be comparable to that of SIRTF's surveys at 24 and $70 \mu \mathrm{m}$. At present it seems quite unclear what, if anything, is the connection between the distant sources seen at these two extremes. Are they the same objects? Are they seen at the same cosmic epoch? SIRTF's sensitivity is well-suited to this purpose. Figure 5 shows, for example, the SED of the nearest ultraluminous infrared galaxy, Arp 220, as it would appear at redshift $z=1$, and that of a $\sim 15 \times$ more luminous object which might be found at $z=3$. Note that both of these objects 
lie well above the SIRTF deep survey levels at all wavelengths, and both are also in the brightness range $\sim 1 \mathrm{mJy}$ reachable with SCUBA at $850 \mu \mathrm{m}$.

Central to this linkage will be the positional accuracy of the SIRTF data products, which will have $\sim 1.4^{\prime \prime} 1-\sigma$ radial absolute uncertainty; this can easily be improved at shorter wavelengths by using the 2MASS catalog and the many stars which will appear in every IRAC frame. This should make it possible to bootstrap from visible/near-infrared sources, to IRAC deep survey frames, to MIPS deep survey frames, and to the likely counterparts of the SCUBA sources.

Because of the concentration of its survey observations in areas well studied at other wavelengths, the scientific yield from the SIRTF surveys will go far beyond the characterization of the infrared sky to include: 1) linking near-infrared visible sources to the SCUBA sources (as discussed above); 2) exploration of the connection between infrared and X-ray emission from the most distant objects; 3 ) establishing a very broad baseline for template spectral energy distributions and photometric redshifts; and 4) contributing to a very large multi-spectral data base which can be used to develop physical models of infrared-bright sources and of their evolution with cosmic time.

A very important result of the SIRTF surveys will be a determination of how much of the star formation history of the Universe is "hidden" in the infrared. The well-known Madau plot of star formation rate per unit mass as a function of cosmic epoch was initially based entirely on ultraviolet to near-infrared observations. It thus omitted any star formation activity which lay concealed within dusty, obscured regions of the type known to harbor young stars throughout the local Universe. Subsequent revisions of this important plot have applied large and uncertain corrections to include this contribution and generally indicate a much higher star formation rate at earlier epochs than did the initial Madau plot. SIRTF's observations, in particular when directly compared to the ultraviolet-to-near infrared observations used for the initial estimates, can clarify this uncertain situation. (SIRTF will observe the HDF-N field from which portions of the original Madau plot were derived, thus making this comparison a very direct one.) One important approach would be SIRTF observations of "dropout" sources - and other distant galaxies - used to characterize star formation at different epochs to determine directly the amount of hidden star formation occuring in these objects. Of equal importance will be comparison of SIRTF observations to ultraviolet-to-near infrared images of the same fields to determine how many objects are seen in the infrared without shorter wavelength counterparts. These could be the tip of the iceberg of an extragalactic population which would have been missed entirely in earlier observations but might account for a substantial fraction of the star formation activity over cosmic time.

\subsection{Infrared Spectra of Infrared-Luminous Galaxies to $z \sim 2$ and Beyond}

The results from ISO (Sturm et al. 2000) show that the 5-40 $\mu \mathrm{m}$ region is home to numerous spectral features - both gas phase emission lines and broader dust phase emission features - that provide both robust redshift indicators and diagnostic information about the ultimate energy sources in infrared galaxies. These include the aromatic hydrocarbon features in the 6-12 $\mu \mathrm{m}$ region and a large family of neon emission lines [from ionization states ranging from doubly- 


\section{Arp 220}

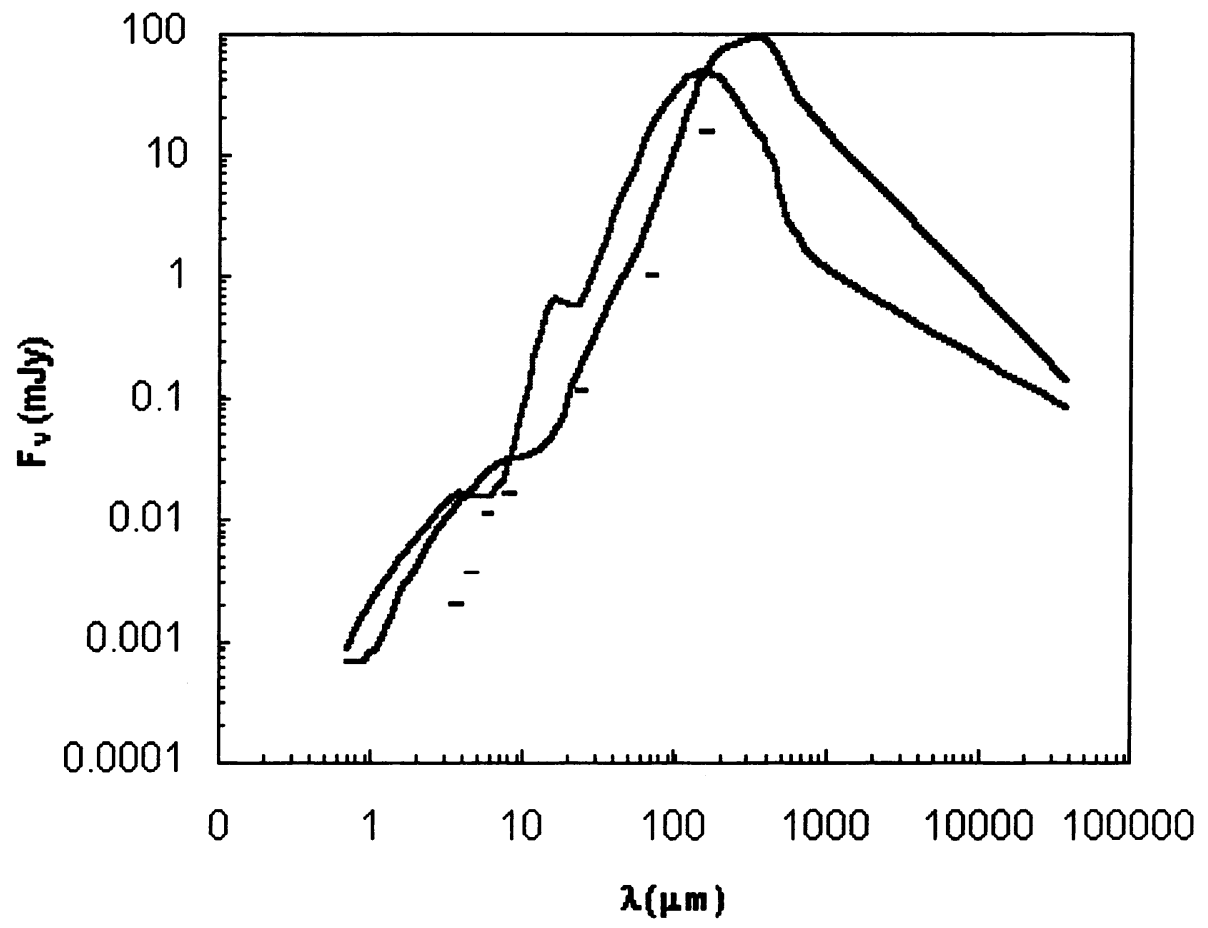

$-z=1$

$-z=3(1+z)^{2}$ Luminosity Evolution

- "Deep" Detection Limits (5-sigma)

Figure 5. The predicted spectral energy distributions of Arp 220 at a redshift $z=1$, and [curve peaking at longer wavelengths] of a $\sim 16 \times$ more luminous object at $z=3$. For reference, the luminosity of Arp 220 is $\sim 3 \times 10^{12} \mathrm{~L}_{\odot}$. The short horizontal bars show the 5- $\sigma$ detection limits to be reached in the SIRTF "Deep" survey (Table 2). 

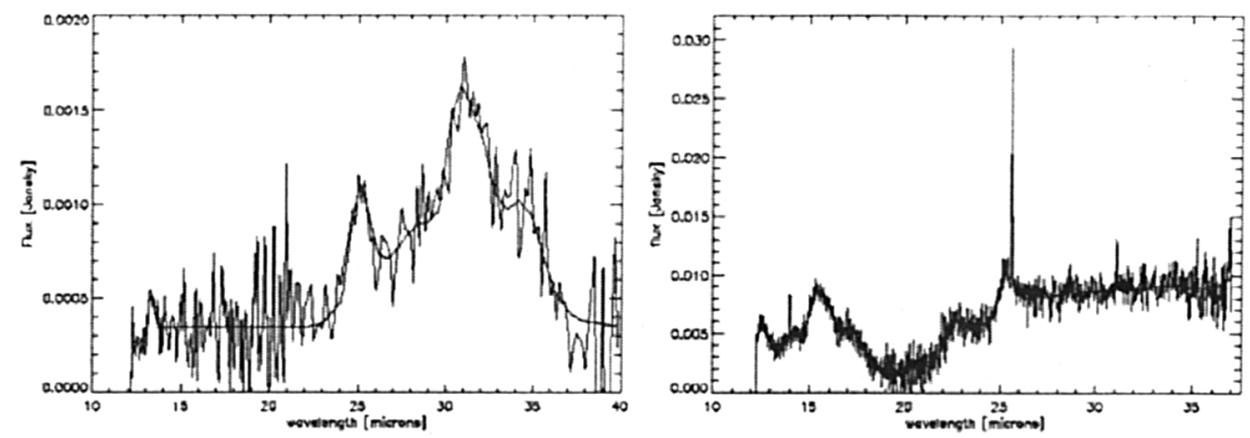

Figure 6. Simulated IRS followup spectra of distant galaxies which might be detected in the GTO "Wide" Survey (Table 2). The model galaxy has a composite Mkn $231+$ M82 spectrum. The left panel shows a low-resolution spectrum of the galaxy at $z=3$; note that the $7.7 \mu \mathrm{m}$ aromatic hydrogen feature is seen at $\sim 31 \mu \mathrm{m}$. At this redshift the galaxy has a luminosity of $\sim 3 \times 10^{13} \mathrm{~L}_{\odot}$. The right panel shows a high resolution IRS spectrum of the galaxy at $z=1$; the $12.8 \mu \mathrm{m}$ [Ne II] line and the $15.6 \mu \mathrm{m}$ [Ne III] line are seen at double their rest wavelengths. The IRS integration time for each panel is $1000 \mathrm{~s}$. (Figure courtesy of D. Weedman.)

to quintuply-ionized]. As an example of what SIRTF can do, the "Wide" survey shown in Table 2 is in fact tuned to the sensitivity of the IRS and should yield a data base of thousands of infrared-emitting galaxies for spectral study from SIRTF.

Figure 6 shows that such SIRTF measurements can detect both the gas phase and dust phase emission features familiar in the nearby Universe in luminous galaxies at redshifts as great as 3 . In doing this, SIRTF can also study how the infrared spectra vary with the metallicity of the galaxy, which will be an important constraint on attempts to synthesize the infrared background. Several GTO programs are devoted directly to this metallicity question by studying the infrared spectra of known low-metallicity galaxies in the nearby Universe. In addition, the mid-infrared spectra to be obtained by SIRTF include enough emission lines that a metallicity determination should be possible from SIRTF's observations alone.

SIRTF can obtain spectra of infrared-bright sources out to $z>2$, encompassing the redshift range of the sources thought to produce the bulk of the recently-identified far infrared background. With these spectral measurements SIRTF can verify the template spectra and spectral energy distributions used in modeling the infrared background and search for evolution of the spectral characteristics of infrared sources - perhaps related to metallicity variations as indicated above - with cosmic time.

The SIRTF spectra will be an extremely important component of the effort described in the previous section to use multiwavelength observations to determine the physical nature of infrared bright sources. For sources which emit predominantly in the infrared, only infrared observations are sure to penetrate 


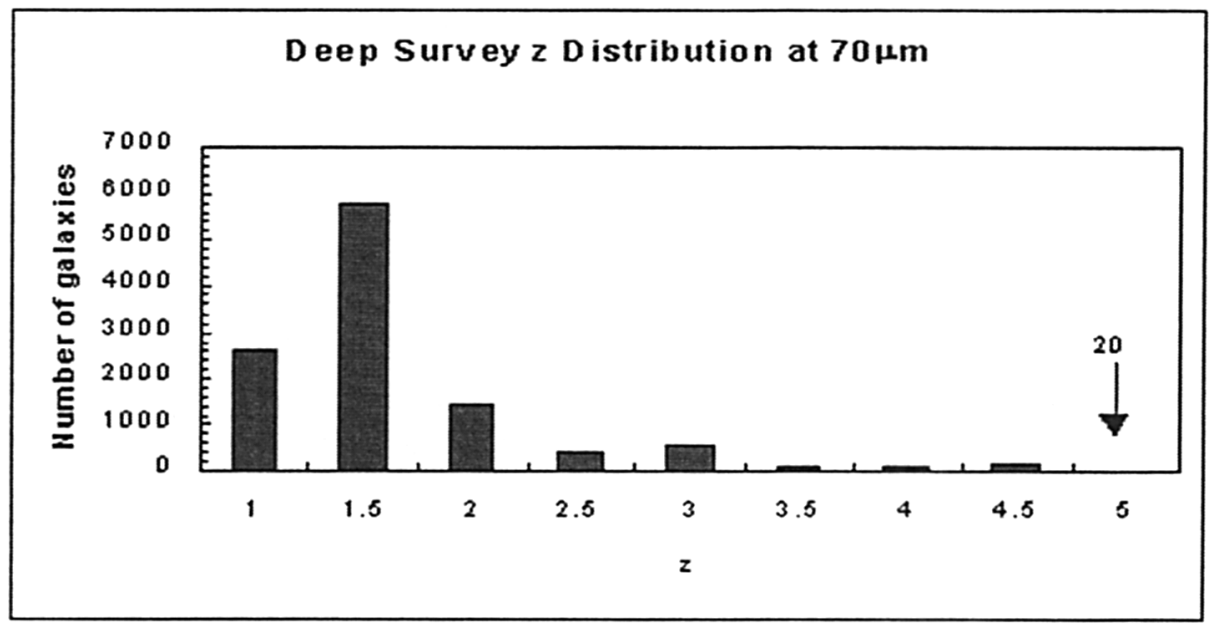

Figure 7. The predicted distribution with redshift of the galaxies detected at $70 \mu \mathrm{m}$ in the GTO "Deep" Survey (Table 2), based on the model spectral energy distributions of Devriendt, Guiderdoni, \& Sadat (1999) and pure luminosity evolution varying as $(1+z)^{2}$.

into their perhaps highly obscured interiors to study conditions in the immediate vicinity of their hidden power sources. Here the broad range of ionization states accessible to SIRTF, ranging from NeII and ArII at the low ionization end to $\mathrm{NeVI}$ and OIV at the high end, will allow SIRTF observations to determine the amounts of star formation and non-thermal activity powering various objects. In addition, SIRTF can study the relative contributions of these two processes as a function of redshift, perhaps out to $z>3$ and see whether the relative strength of the aromatic hydrocarbon emission feature at $7.7 \mu \mathrm{m}$ is as robust an indicator of starburst activity at high redshift as it appears to be locally (Lutz et al. 1998). As another example, interacting galaxies may evolve through a luminous starburst to a state of very high central density which ends up by feeding matter into a [perhaps pre-existing] black hole. SIRTF's observations should be able to delineate this evolution by tracing the relative amounts of thermallyand non-thermally excited emission from interacting pairs of galaxies of various characteristics. Finally, given that there are galaxies known with $L_{i r} / L_{v} \sim 100$, there is every reason to expect other galaxies to be found with $L_{i r} / L_{v}>1000$ infrared-emitting objects with no visible wavelength counterparts. Such objects may be identified in SIRTF surveys, and SIRTF spectroscopy could be our only immediately available technique for determining the physical nature of such a new and exciting population.

\subsection{Finding the Needle in the Haystack}

The most extreme objects - in the current context perhaps the most distant and/or the most luminous - will always be of interest as they provide the most severe tests of our physical understanding. Thus, for example, discovery of 
a substantial population of dusty, massive, and luminous galaxies [as opposed to quasars] at $z>5$ might require reassessment of some ideas about galaxy formation in the early Universe. With its large area surveys, high sensitivity, and substantial diagnostic capability, SIRTF has the potential to provide this type of discovery. In Figure 7, for example, we present the expected distribution with redshift of the galaxies to be found in the Deep survey presented in Table 2. Note that $\sim 10,000$ galaxies with $z<1$ will also be detected. This model predicts a tail in the distribution with a few tenths of a percent of the galaxies lying beyond $z>5$. Although these unusual objects might not be the first ones to be culled from the data base, the SIRTF survey data, again in combination with the correlated observations across the spectrum, will support the type of systematic analysis which should eventually lead to the identification of such sources, if they exist. Additionally, in the sense of discovery phase space, SIRTF represents a great advance in our capability to explore the Universe at infrared wavelengths. Thus, there is every reason to anticipate discoveries from SIRTF perhaps much more luminous objects and much higher redshifts than previously probed - that might dramatically alter our perception of the early evolution of stars and galaxies.

\section{How You can Use SIRTF - the General Observer Opportunities}

It is hoped that this paper has whetted the readers' appetites in anticipation of carrying out their own programs with SIRTF. Beyond the Legacy Science program, the community will be given multiple opportunities to propose General Observer projects and/or Archival Research with SIRTF. The first call for General Observer proposals will be issued in May, 2002, with proposals due in November, 2002. The first General Observer observations would take place in April, 2003, on this schedule. Subsequent opportunities for General Observer and Archival Research proposals will occur on approximately one-year centers. Over $75 \%$ of the observing time on SIRTF will be allocated to the community through the combined General Observer and Legacy programs, and the community may also apply for Director's Discretionary Time under exceptional circumstances.

The General Observer program - from solicitation of proposals through distribution of the processed data to the observers - will be run through the SIRTF Science Center (SSC) on the Caltech campus. All interested parties are invited to stay abreast of the progress of SIRTF and the General Observer and Archival Research opportunities by monitoring the SIRTF Web Site: sirtf.caltech.edu.

Acknowledgments. Portions of this work were carried out at the Jet Propulsion Laboratory, California Institute of Technology, operated under a contract with the National Aeronautics and Space Administration

\section{References}

Dermott, S. F., Jayaraman, S., Xu, Y. L., Gustafson, B. A. S., \& Liou, J. C. 1994, Nature, 369, 719

Devriendt, J. E. G., Guiderdoni, B., \& Sadat, R. 1999, A\&A, 350, 381 
Dwek, E., \& Arendt, R. G. 1998, ApJ, 508, L9

Fanson, J. L., Fazio, G. G., Houck, J. R., Kelly, T., Rieke, G. H., Tenerelli, D. J., \& Whitten, M. 1998, Proc. SPIE, 3356, 478

Franceschini, A., Toffolatti, L., Mazzei, P., Danese, L., \& De Zotti, G. 1991, A\&A Supp. Ser., 89, 285.

Gorjian, V., Wright, E. L., \& Chary, R. R. 2000, ApJ, 536, 550

Hogg, D. W., Neugebauer, G., Cohen, J. G., Dickinson, M., Djorgovski, S. G., Matthews, K., \& Soifer, B. T. 2000, AJ, 119, 1519

Jayaraman, S., Dermott, S., \& Werner, M. 1996, in ASP Conf. Ser. Vol. 104, Physics, Chemistry, and Dynamics of Interplanetary Dust, ed. B.Gustafson \& M. Hanner (San Francisco: ASP), 159

Lutz, D., Spoon, H. W. W., Rigopoulou, D., Moorwood, A. F. M., \& Genzel, R. 1998, ApJ, 505, 105.

Reach, W. T., Franz, B. A., Weiland, J. L., Hauser, M. G., Kelsall, T. N., Wright, E. L., Rawley, G., Stemwedel, S. W., \& Spiesman, W. J. 1995, Nature, 374, 521

Sturm, E., Lutz, D., Tran, D., Feuchtgruber, H., Genzel, R., Kunze, D., Moorwood, A. F. M., \& Thornley, M. D. 2000, A\&A, 358, 481

Tan, J. C., Silk, J., \& Balland, C. 1999, ApJ, 522, 579

Wright, E. L. 2000, ApJ, submitted

Wright, E. L., Werner, M., \& Rieke, G. 1995, in AIP Conf. Proc. 348, Unveiling the Cosmic Infrared Background, ed. E. Dwek (New York: AIP), 278 